\title{
Closure of Pfannenstiel skin incisions in cesarean sections: comparison of wound outcomes with interrupted mattress vs. subcuticular suture
}

\author{
Anjali Choudhary*, Neeta Bansal, Priyanka Chaudhari
} Department of Obstetrics and Gynecology, Shree Guru Ram Rai Institute of Medical and Health Services, Dehradun,
Uttarakhand, India

Received: 29 April 2017

Accepted: 29 May 2017

*Correspondence:

Dr. Anjali Choudhary,

E-mail: mcgsunil@gmail.com

Copyright: (c) the author(s), publisher and licensee Medip Academy. This is an open-access article distributed under the terms of the Creative Commons Attribution Non-Commercial License, which permits unrestricted non-commercial use, distribution, and reproduction in any medium, provided the original work is properly cited.

\begin{abstract}
Background: Cesarean section is by far the commonest surgery performed worldwide. Most of these surgeries are performed by Pfannenstiel incision. Various techniques and material have been tried for skin closure in these surgeries. Each method has its own advantages and disadvantages. The objective of the study was to compare wound outcomes in Pfannenstiel incisions closed in interrupted mattress sutures and subcuticular suture with absorbable material, in a prospective study of cesarean deliveries.

Methods: It was studied that 104 women who had cesarean sections with Pfannenstiel incision prospectively for wound outcomes in terms of wound complications (hematoma formation, infection, nonunion, dehiscence and need for resuturing), pain and cosmetic appearance in two groups. One with absorbable subcuticular sutures, another with interrupted mattress suture.

Results: Women who had subcuticular stitches had less postoperative pain, better wound outcomes, faster recovery, early discharge from hospital and cosmetically superior scars as compared with interrupted mattress sutures.

Conclusions: While search for the best method and material for skin closure continues, the subcuticular suture with an absorbable material seems to leave a cosmetically better scar with lesser wound complications.
\end{abstract}

Keywords: Interrupted mattress stitch, Pfannenstiel incision, Skin closure, Subcuticular suture, Wound outcome

\section{INTRODUCTION}

Cesarean section is by far the commonest surgery performed worldwide. Most of these surgeries are performed by Pfannenstiel incision. Pfannenstiel incision was first described and performed by Hermann Pfannenstiel in $1897^{1}$. This is a low transverse suprapubic incision which involves dissection of rectus muscle from overlying rectus sheath, after incising skin and rectus sheath. Since its introduction it has rapidly become popular and is the incision of choice in many obstetric and gynecologic surgeries. Most of cesarean section nowadays is performed by this incision. ${ }^{2,3}$ It offers adequate exposure of pelvic viscera and leaves a cosmetically acceptable scar. When properly placed it generally concealed by re-growth of pubic hair-hence the name bikini incision. It has become more popular in recent times in younger women for better cosmetic results Skin incisions need closure to restore function, reduce pain and bleeding. Various techniques and materials have been tried for skin closure for these surgeries. Each method has its own advantages and disadvantages.

Wound healing in a non-contaminated surgical wound with edges in apposition takes place by migration of new epithelial skin cells from the edges of the incision. Basic 
skin union can be seen by 48 hours post-surgery, whereas complete union is visible by seventh or eighth postoperative day. Skin healing with restoration of epithelium without fibrosis takes place by primary intention, in the absence of infection, hematoma formation or other wound complications. ${ }^{4}$ Main aim of the surgical suturing is to bring the skin edges together, without tension and the healing would take place naturally. The goals of wound closure include obliteration of dead space, even distribution of tension along deep suture lines, and maintenance of tensile strength across the wound. ${ }^{5}$

Many techniques and suture materials have been tried to achieve closure and restore the structural and functional anatomy of skin. Suture materials that have been tried are natural and synthetic materials, which are absorbable and non-absorbable. ${ }^{6}$ Various methods used to close such wounds are interrupted mattress sutures, staples, adhesive tapes and absorbable or non-absorbable and subcuticular stitches with absorbable and non-absorbable materials. ${ }^{7}$

Material and technique used for skin closure influences the quality of wound outcome, in terms of post-operative pain, induration, infection, healing, cosmetic appearance and wound acceptance by patient. $^{8}$ A good suture material is the one which causes the least foreign body reaction and inflammation. A good surgical technique is the one that eliminates dead space in subcutaneous tissue and approximates edges without tension. ${ }^{6,7}$

Different suture materials produce varying degrees of tissue reaction, specifically inflammation. Significant inflammation reduces the resistance to infection and delays the onset of wound healing. The type of material and size of the suture are thought to be the major factors contributing to this reaction. Natural absorbable materials are absorbed by proteolysis, which causes a prominent inflammatory response, while synthetic absorbable materials are absorbed by hydrolysis, which produces a minimal tissue reaction. ${ }^{9}$ Best ways to close skin incisions for optimal results are still under experimentation. ${ }^{5,10}$ There is a need to identify the right method and suture material to provide the best wound outcomes. The method should have less postoperative morbidity, help faster recovery and be cost effective at the same time.

A subcuticular stitching involves repairing the subcuticular or the epidermal tissue in a running suture. This technique brings the skin edges together, reduces the dead space and spares the skin from the trauma of the needle entry. Earlier method of closing thicker tissues was piercing a larger needle penetrating deep enough for a larger tissue to be gathered together and tied tightly to compress everything together to cause haemostasis- as in the interrupted mattress stitch. ${ }^{7}$

A more modern approach recognizes that skin is made of layers of different tissue with properties which may be better served with different threads and stitches. The sub cutis, also called the hypoderm, is the innermost layer of skin. It is made up of several layers of connective tissue which has a good tensile strength. A subcuticular suture repairs this skin layer first. Suturing of the outer epidermis follows, by using thinner suture material with small but sharp needles, often using techniques to minimize scarring. The resultant scar is linear, thin and cosmetically more acceptable. ${ }^{6}$ The advantage of this technique is the relatively rapid speed with which it can be placed, with least trauma to skin. If an absorbable suture is used it can be left in place eliminating the need for suture removal, facilitating an early discharge from the hospital. A subcuticular stitch in a Pfannenstiel incision runs along the natural skin lines hence improves and hastens the wound healing. Since it avoids piercing the skin directly, which has maximum nerve endings, the post-operative pain is less, allowing early ambulation and faster recovery.

Interrupted vertical mattress stitch involves piercing the skin at four points at the same level for a single stitch, gathers a good amount of subcuticular tissue and fat, in a tight stitch and leaves gaps between the stitches to allow drainage of blood and sero-sanguinous discharge of fat necrosis. This type of stitching is time consuming. Improper technique leaves unopposed edges with uneven tension and causes more scarring. ${ }^{6}$ It is also associated with more induration, everted edges, delayed wound healing, infection and wound dissolution. Tight mattress suture might cause strangulation of tissues leading to tissue hypoxia, necrosis and poor wound healing. ${ }^{11}$ The gaps between the stitches allow oozing of blood and serum and delays union of skin edges.

The objective of this effort was to compare wound outcomes in subcuticular suture and interrupted mattress sutures for skin closure in a prospective study of cesarean sections, in terms of cosmoses, pain and wound complications.

\section{METHODS}

It was studied that 104 women who had cesarean deliveries with Pfannenstiel incision prospectively for wound outcomes in terms of wound complications [hematoma formation, infection, nonunion, dehiscence and need for re-suturing], pain and cosmetic appearance in two groups of women. Group one consisted of women with subcuticular sutures with absorbable material (we used ploygalactin (Vicryl) rapid. Group two consisted of women with interrupted mattress stitches with nonabsorbable material (Ethilon).

Women from both groups were questioned regarding amount and intensity of pain and need for analgesics in the early postoperative period. The dressing was removed after forty-eight hours of surgery and the condition of the wound regarding, union, discharge, bleeding, hematoma and indurations were noted. Wound was reassessed at the 
end of one week and after one month for the nature of scar, infection, indurations, pain and cosmoses. Duration of hospital stay and cost of stay was noted. Wound outcome, pain and cosmetic appearance were compared in the both groups after forty-eight hours, seventh day and one month. Women at high risk for wound sepsis like diabetes, history of PROM or chorio-amnionitis, H/o PIH and/or deranged coagulation etc. were excluded from the study.

\section{RESULTS}

A total of 104 women (52 cases in each group.) were included in the study. There was a marginal difference in perception of pain in the immediate post-operative day in both groups. Group 1 with subcuticular suture experienced lesser pain, needed fewer analgesics. After forty-eight hours majority of wounds appeared to be well approximated, with less induration and bleeding edges in group 1. Many women in group 2 had everted, nonaligned skin edged with erythema, ecchymoses, and hardening of skin edges. Some women (19\%) had fresh bleeding or hematoma formation.

Women with subcuticular suture (group 1) had clean well healed united skin, without induration, oozing or infection by end of first week. Since this group had absorbable suture they were discharged early thereby reduced hospital stay and cost. More women from group 2 who had interrupted mattress sutures experienced wound complications like, hematoma, indurations, serosanguinous discharge, sepsis, non-union or dehiscence. These women were discharged from hospital after seventh day.

Table 1: Wound condition after 48 hours.

\begin{tabular}{|lll|}
\hline Parameter & Group 1(52) & Group 2 (52) \\
\hline United edges & $49(94 \%)$ & $39(75 \%)$ \\
\hline Everted edges & $3(5.75)$ & $15(28.84$ \\
\hline Hematoma & $4(7.69 \%)$ & $10(19.23 \%)$ \\
\hline Bleeding & $4(7.69)$ & $14(26.92 \%)$. \\
\hline Induration & $10(19.23 \%)$ & $29(55.76 \%)$ \\
\hline Pain & $12(23 \%)$ & $18(34 \%)$ \\
\hline
\end{tabular}

Table 2: Wound condition after one week.

\begin{tabular}{|lll|}
\hline Parameter & Group 1(52) & Group 2(52) \\
\hline Induration & $10(19.23 \%)$ & $29(55.76 \%)$ \\
\hline Infection & $3(5.69 \%)$ & $20(38.46)$ \\
\hline Mal union & $4(7.69 \%)$ & $23(44.23 \%)$ \\
\hline Resuturing & $3(5.75 \%)$ & $10(19.2 \%)$ \\
\hline
\end{tabular}

About $5.75 \%$ of women from group 1 and $19.7 \%$ of women from group 2 needed re-suturing of the wound. One month follow up of women from group 2 had raised indurated, hypertrophic scars with typical caterpillar appearance. Women from group 1 (subcuticular stitches) had thin linear scar merging well with rest of the skin, without indurations. There was no pain and the scar appeared cosmetically more acceptable, and they had no complaints. Condition of the wound after 48 hours, 1 week and 6 weeks are given in Table 1, 2 and 3 respectively with the graphs 1,2 and 3 .

Table 3: Follow up at six weeks (wound /scar appearance).

\begin{tabular}{|lllll|}
\hline Parameters & Group 1 & $(\%)$ & Group 2 & $(\%)$ \\
\hline Induration & 4 & 7 & 24 & 46.15 \\
\hline Puckering & 0 & 0 & 17 & 32 \\
\hline Thick scar & 3 & 5.7 & 27 & 38 \\
\hline $\begin{array}{l}\text { Cosmetic } \\
\text { appearance }\end{array}$ & 45 & 92 & 25 & $48 \%$ \\
\hline
\end{tabular}

$\%$ cannot be tallied as some women had a combination of parameters present

\section{DISCUSSION}

Pfannensteil incision has become more popular in recent times in younger women for better cosmetic results. Many ways and materials have been tried for skin closure in these incisions

Suture material used for skin closure affects the wound outcome. Although the search for ideal material continues, synthetic absorbable material is emerging as better materials as they provide adequate and longer skin approximation thereby giving better healing and cosmoses. Main aim of any skin closure technique is to produce appropriate approximation and adequate healing, with less pain, scarring and wound complication. The method should be faster, cost effective and have maximum cosmoses and patient satisfaction. ${ }^{3}$

Different materials have been tried with variable results. ${ }^{5}$ An absorbable material provides a temporary support to the skin edges while healing takes place it can be left in place, precluding the necessity of stitch removal. Tumuli et al in their meta-analysis and review of studies comparing subcuticular suture to staple in cesarean sections found that, staple closure is faster to perform but associated with a higher risk of wound complications. ${ }^{12}$ In a similar study Clay et al analyzed randomized control trials for subcuticular versus staples for closure in csections concluded that subcuticular stitch was beneficial as compared to staples in terms of wound outcome an observation similar to our findings. ${ }^{13}$

Mackerel AD Bergehella, VL Larsen ML in their systematic review of Cochrane database on techniques and materials for closure in cesarean sections, compared non -absorbable staples to absorbable subcutaneous suture found that staples are associated with increased risk of skin separation and re-suturing. ${ }^{7,2}$ In our observation subcuticular suture with absorbable material offered better skin approximation and healing than the interrupted suture, which needed re-suturing more often. 
In another prospective randomized trial performed by Brown JK et al where they compared subcuticular suture to skin adhesive, observed that there was no difference in cosmetic outcome in subcuticular suture and skin adhesive and subcuticular stitch provided adequate skin apposition for proper healing. Skin adhesive on the other hand is more expensive to suture though it reduces operating time. ${ }^{9}$ It was found that subcuticular suture is not only cost effective but it also saves operating time when compared with interrupted mattress stitches.

In a similar study JD Kolt used adhesive surgical tape with the absorbable continuous subcuticular suture and concluded that use of continuous absorbable subcuticular suture allows accurate skin edge approximation, which along with adhesive tape increases the safety margin. The combination closure has a slightly better cosmetic result than with suture less tape alone or subcuticular stitch alone. ${ }^{10}$

Absorbable subcuticular sutures when placed into subcutenous tissues eliminate the dead space, reducing potential spaces for collection of blood and serum. It brings the edges closure for tension free apposition of skin. When epidermis is closed it avoids direct needle contact with skin surface, allowing better healing. Synthetic monofilament material has less tissue reaction hence are better for skin closure. Mohan Kudur et al in their extensive description of suture materials and suturing techniques praised the subcuticular running suture as a fast and useful technique with enhanced cosmetic results. ${ }^{6}$

In present study it was observed that subcuticular absorbable suture is superior to interrupted mattress stitches with regard to many variables for the wound outcome. Time taken for skin closure was less; approximation was better and without tension. Women were discharged earlier since there was no need for suture removal.

Very few women required re-suturing as compared with women with interrupted stitches. In a large systematic review of Cochrane database, Guruswamy et al compared, continuous versus interrupted skin sutures for non-obstetric surgeries found that Superficial wound dehiscence may be reduced by using continuous subcuticular sutures. This observation is in agreement with our findings, we observed lesser instances of wound gaping with subcuticular stitch. ${ }^{8}$

Aesthetics is an important aspect of a good surgery. Abdominal closure after surgery should not only aim at restoring anatomy and function but also look neat, appealing and leave cosmetically acceptable scars. We found running subcuticular suture with synthetic absorbable material for closure of Pfannenstiel incisions have all these advantages, are faster to perform, leave a better scar with less complications, allow early ambulation and shorter hospital stay.

\section{CONCLUSION}

Wound outcome and scar appearance after surgery with Pfannenstiel incision largely depends upon the material and the technique used for skin closure. There is a continual search for 'ideal' way to close surgical wounds with the best possible outcome. In our observation subcuticular stitches with absorbable synthetic material, appear to be superior to the interrupted mattress stitches to close Pfannenstiel incisions with regard to wound outcome and cosmetic appearance.

Funding: No funding sources

Conflict of interest: None declared

Ethical approval: The study was approved by the Institutional Ethics Committee

\section{REFERENCES}

1. Wylie BJ, Gilbert S, Landon MB, Spong CY, Rouse DJ, Leveno KJ, et al. Comparison of transverse and vertical skin incision for emergency cesarean delivery. Obstet Gynecol. 2010;115(6):1134-40.

2. Figueroa D, Jauk VC, Szychowski JM, Garner R, Biggio JR, Andrews WW et al. Surgical staples compared with subcuticular suture for skin closure after cesarean delivery: a randomized controlled trial. Obstet Gynecol. 2013;121(1):33-8.

3. Altman AD, Allen VM, McNeil SA, Dempster J. Pfannenstiel incision closure: a review of current skin closure techniques. J Obstet Gynecol Canada. 2009;31(6):514-20.

4. Walter JB, Israel MS. Wound healing, Textbook of general pathlogy, $6^{\text {th }}$ ed. Ch.9:124-5.

5. Aslam A, Eshan A. Comparison of suture material and technique of closure of subcutaneous fat and skin in cesarean section. N Am J Med Sci. 2011;3(2):856.

6. Kudur MH, Pai SB, Sripathi H, Prabhu S. Sutures and suturing techniques in skin closure. Indian $\mathbf{J}$ Dermatol Venereol Leprol. 2009;75:425-34.

7. Mackeen AD, Berghella V, Larsen ML, Techniques and materials for skin closure in caesarean section. Cochrane Database Syst Rev. 2012;11:CD003577.

8. Gruswamy KS, Toon CD, Allen VB, Davidson BR. Continous versus intrupted sutures for non-obstetric surgery. Cochrane Database Syst Rev. 2014;142:CD010365.

9. Brown JK, Campbell BT, Dronogowski RA, Alderman AK, Giger JD, Teitlbaum DH, et al. A prospective, randomized comparison of skin adhesive and subcuticular suture for closure of pediatric hernia incisions: cost and cosmetic considerations. J Pediatr Surg. 2009;44(7):1418-22.

10. Kolt JD. Use of adhesive surgical tape with the absorbable continuous subcuticular suture. ANZ J Surg. 2003;73(8):626-9.

11. Margarate Terhune, Materials for Wound Closure, Medscape. 2012. 
12. Tuuli MG Rampersad RM, Carbone JF, Stamilio D, Macones GA, Odibo AO. Staples compared with subcuticular suture for skin closure after cesarean delivery: a systemic review and meta analysis Obstet Gynecol. 2011;117(3):682-90.

13. Clay FS, Walsh CA, Walsh SR. Staples vs. subcuticular sutures for skin closure at cesarean delivery: a metaanalysis of randomized controlled trials. Am J Obstetric Gynecol. 2011;204(5):378-83.

Cite this article as: Choudhary A, Bansal N,

Chaudhari P. Closure of pfannenstiel skin incisions in cesarean sections: comparison of wound outcomes with interrupted mattress vs. subcuticular suture. Int J Reprod Contracept Obstet Gynecol 2017;6:2964-8. 